\title{
The Mathematical Model and the Simulation Modelling Algoritm of the Multitiered Mechanical System
}

\section{Demin Anatoliy, Kovalev Ivan}

Dept. of Optical Digital Systems and Technologies, The St. Petersburg National Research University of Information Technologies, Saint Petersburg, RUSSIAN FEDERATION

\section{(LOMO PLC)}

Modern mechanical systems are usually haven complex spatial and multitiered structure. The important stage of the design of such systems is to estimate its behavior in the dynamics which can be obtained by computer simulation.

Figure 1 below shows a multi-mechanical control system of the supervisory system, including a mobile base (deck of a ship) and the observation device in gimbal suspension. The feature of this system is that the mass centers of components of the system are separated in space.

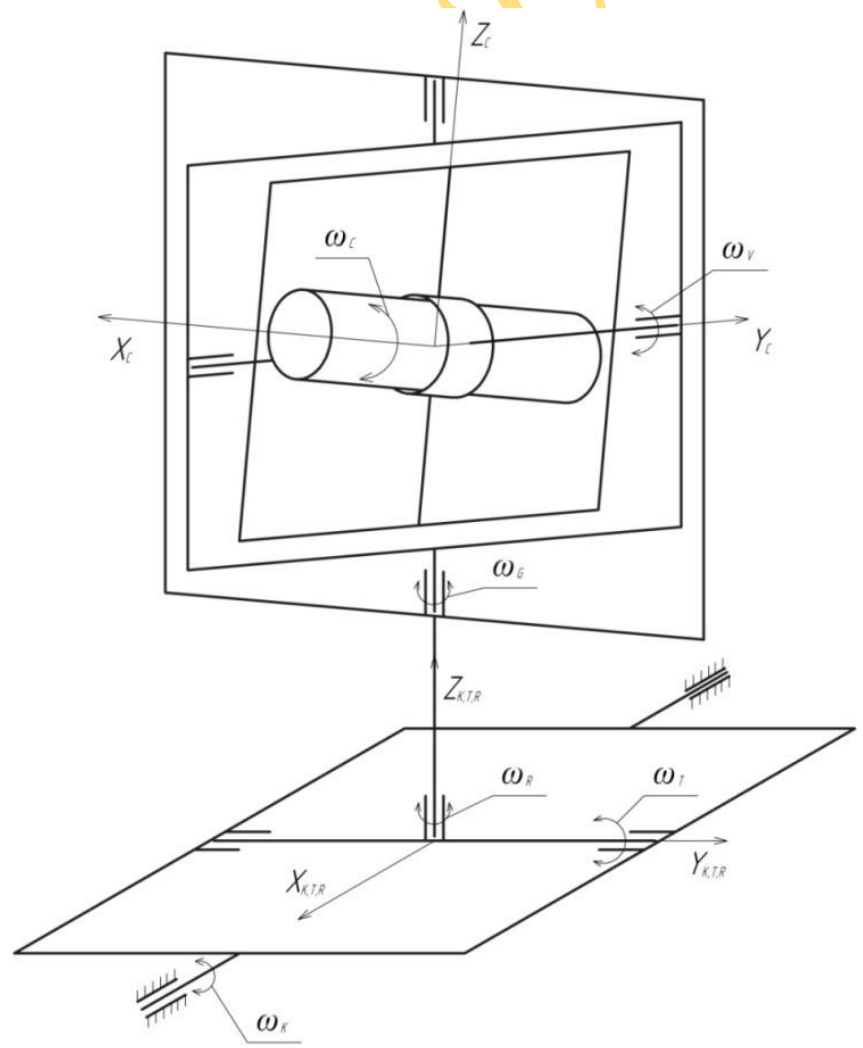

Fig. 1 Multitiered mechanical control system of the supervisory system. 
Figure 1 uses the following notations: the first three components $-K, T, R-$ simulate the rotation of the vessel relative to three orthogonal axes, so that, $\omega_{\mathrm{K}}$ - the angular velocity of rotation of the deck at the angle of heel, $\omega_{\mathrm{T}}$ - the angular velocity of rotation of the deck at the corner of yaw, $\omega_{\mathrm{R}}$ - the angular velocity of rotation of the deck at the corner of pitch attitude. The poles of these components are the same and are in the center of the ship rolling. The second three components $-\mathrm{G}, \mathrm{V}, \mathrm{C}$ - simulate the optical device rotation so that, $\omega_{\mathrm{G}}$ - the angular velocity of the optical device rotation by horizontal angle, $\omega_{\mathrm{V}}-$ the angular velocity of the rotation of the optical device by vertical angle, $\omega_{C}$-the angular velocity of the optical device rotation by angle of compensation.

The simplest and most effective way to set up the equations of such systems with geometric constraints on the location of its points (not free holonomic system) is based on the representation of their relatively generalized forces determined by the energy of the system, and generalized coordinates. The equations of motion in this case as the Lagrange equations of the 2 nd kind can be represented in vector-matrix form:

$$
(A \dot{q})=Q+0,5 D \dot{q}=Q+\frac{1}{2}\left[\frac{\partial\left(a_{i j}^{j} \dot{q}_{i j}\right)}{\partial q}\right]_{j=1}^{n} \times \dot{q}
$$

where $A$ - the matrix of derivatives of the kinetic energy by coordinates; $Q$ generalized forces matrix; $q$ - generalized coordinates matrix $(i=1,2, \ldots) ;$,$D - partial$ derivatives matrix; $\mathrm{n}$ - the number of degrees of freedom; $a_{\mathrm{ij}}$ - coefficients of inertia.

The advantage of this representation is its generality and uniform of constructing the equations. However, due to the fact that the presentation of (1) is complex in terms of the spatial orientation of the system and analysis of its constituent parts, appears difficulty in simulation modeling. In order to simplify the procedure of building up the equations, we take the concept of quasi-velocities, i.e. we'll carry out the linearization of the generalized velocities. Such a simplification allows a mathematical model of motion of the mechanical part of the system in a form suitable for its partial analysis. In this case the stage of the withdrawal of differential equations for the dynamics of the body can be omitted. In this way we can present the mechanical multitiered control system of the optical device (see fig.1) in form of the graphic-vector description as shown in Figure 2, where the following notations are used : $\mathrm{O}$ - the center of the earth rotation; $\mathrm{V}_{\mathrm{K}}$ - the linear speed of the ship; $\mathrm{K}$, $T, R, G, V, C$ - poles of the relevant parts of the system; $R_{K, T, R, G, V, C}$ - radius vectors of the corresponding poles; $\mathrm{R}_{\mathrm{C}}$ - radius vector of the mass center of the corresponding part to the coordinate system associated with this part; $\mathrm{M}_{\mathrm{K}, \mathrm{T}, \mathrm{R}, \mathrm{G}, \mathrm{V}, \mathrm{C}}-$ moments of the forces acting on the relevant body; $\omega_{\mathrm{O}, \mathrm{K}, \mathrm{T}, \mathrm{R}, \mathrm{G}, \mathrm{V}, \mathrm{C}}$ - angular velocity vector of the relevant parts; $\mathrm{D}_{\mathrm{T}, \mathrm{R}, \mathrm{G}, \mathrm{V}, \mathrm{C}}-$ the poles of the drive units of the corresponding parts; $R_{D}$ - radius vectors of drive unit poles to the coordinate system associated with the respective parts; $\omega_{\mathrm{D}}$ - angular velocities of the respective drive units; $R_{C D}$ - radius vectors of the moments of the forces corresponding to the appropriate drive units; $\mathrm{M}_{\mathrm{D}}$ - moments of forces of corresponding drive units; $\gamma_{T, R, G, V, C}-$ angles of bodies rotation under the action of the corresponding drive units. 


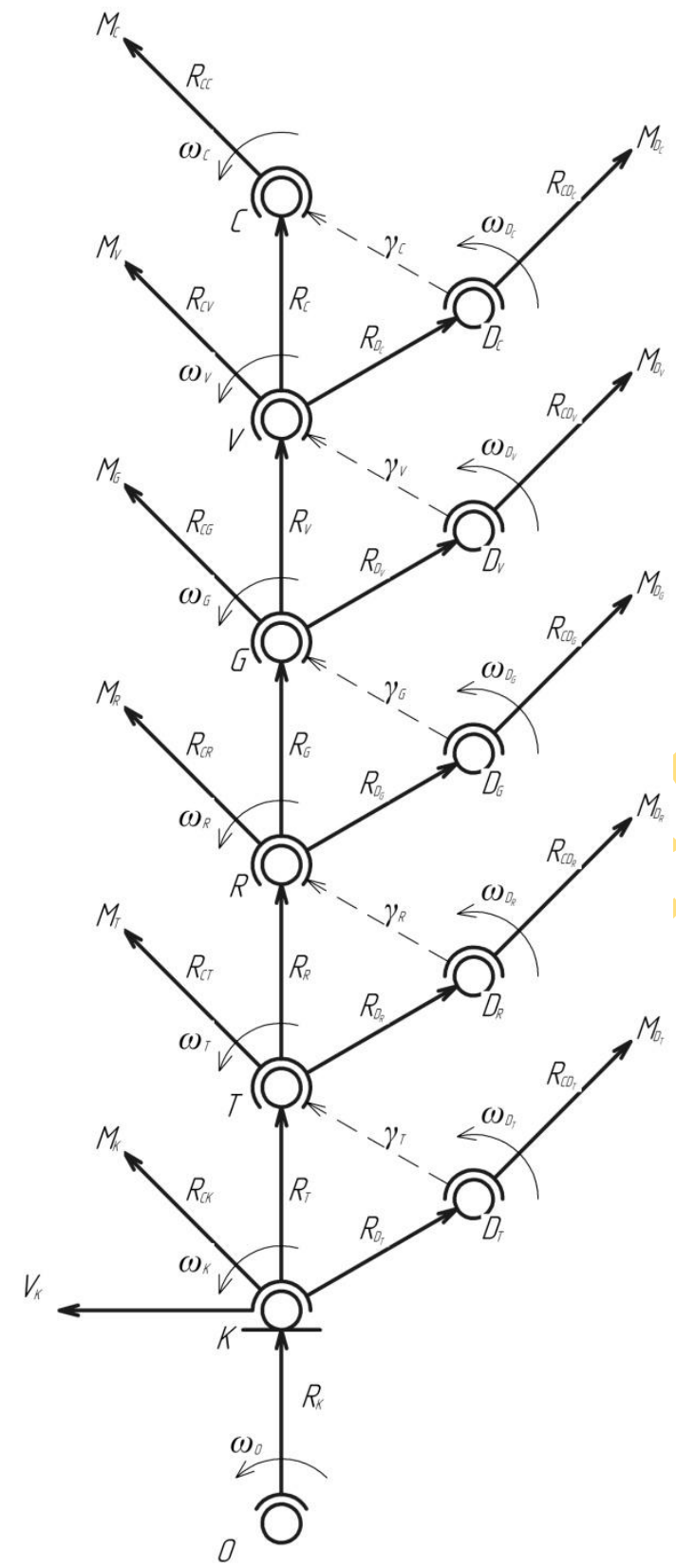

Fig.2 Graphic-vector description of the multitiered mechanical system of optical device control

A mathematical model of motion of the mechanical multitiered system can be made on the basis of: the law of conservation of energy; virtual work in conjunction with the principle d'Alembert; Newton's and Euler's equations; Lagrange equations; Hamilton's principle. According to this ratio as the initial set-known equations of the free body motion in a vector form:

$\left\{\begin{array}{l}m_{0}\left[\bar{W}_{0}+\bar{\omega}_{0} \times \bar{r}_{0}+\bar{\omega}_{0} \times\left(\bar{\omega}_{0} \times \bar{r}_{0}\right)\right]: \\ J_{0} \bar{\omega}_{0}+\bar{\omega}_{0} \times\left(J_{0} \bar{\omega}_{0}\right)+m_{0} \bar{r}_{0} \times \bar{W}_{0}=\bar{\Lambda} \\ \bar{W}_{0}=\dot{\bar{V}}_{0}+\bar{\omega}_{0} \times \bar{V}_{0},\end{array}\right.$ where $\mathrm{m}_{0}$ и $\mathrm{J}_{0}$ - mass and inertia tensor given to the top of the coordinate system fixed to the body $\mathrm{X}_{0} \mathrm{Y}_{0} \mathrm{Z}_{0} ; \bar{F}_{0}$ и $\bar{M}_{0}$ - the main vectors of external forces and moments of external forces applied to the body according to the coordinate system $\mathrm{X}_{0} \mathrm{Y}_{0} \mathrm{Z}_{0} ; \bar{\omega}_{0}$ и $\bar{V}_{0}$ - angular and linear velocity of top of the coordinate system $\mathrm{X}_{0} \mathrm{Y}_{0} \mathrm{Z}_{0} ; \bar{r}_{0}$ - radius vector of the body's mass center; $\bar{W}_{0}$ - acceleration vector of the coordinate system $\mathrm{X}_{0} \mathrm{Y}_{0} \mathrm{Z}_{0}$.

The first equation expresses the theorem quantity of motion (equation of motion of the mass center). The second equation expresses the theorem quantity of motion relative to the coordinate system $\mathrm{X}_{0} \mathrm{Y}_{0} \mathrm{Z}_{0}$ (equation of "rotation").

Any mechanical system can be decomposed into a finite set of solid bodies $\mathrm{S}_{\mathrm{i}}(\mathrm{i}=\mathrm{n}, \ldots, \mathrm{N} ; \quad \mathrm{n}=0,1, \ldots)$. The position of each body $S_{i}$ will be determined relative to the body $S_{i-1}$ of this set, and the position and movement of the body $S_{0}$ will be determined relative to the inertial coordinate system XYZ. We will also assume that the body $S_{i}$ can move relatively to the body $S_{i-1}$ only under the influence of the force vectors $\bar{F}_{i}^{n}$ from the body $\mathrm{S}_{\mathrm{i}-1}$. In case of motion of the system of solid bodies, the position of the center of inertia $C_{i}$ does not change corresponding to the pole $\mathrm{O}_{i}$, associated with the body $S_{i}$ coordinate system $X_{i} Y_{i} Z_{i}$, but varies in relation to the poles of the coordinate system with the index other than the «i». The position of the center of inertia $C_{O}$ of the body $S_{0}$ relative to the inertial coordinate system $X Y Z$ is defined by the 
radius vector $\boldsymbol{R}_{00}$ (3a), the position of the center of inertia $C_{1}$ of the body $S_{1}$ relative to the $\mathrm{XYZ}$ and $\mathrm{X}_{0} \mathrm{Y}_{0} \mathrm{Z}_{0}$ is determined by the radius vector $\boldsymbol{R}_{\mathbf{0 1}} \mathbf{( 3 \sigma )}$ ), and in general case (3c):

$$
\begin{gathered}
\bar{R}_{00}=\bar{R}_{0}+\bar{R}_{C 0}, \\
\bar{R}_{00}+\bar{R}_{1}+\bar{R}_{C 1}=\bar{R}_{0}+\bar{R}_{C 0}+\bar{R}_{1}+\bar{R}_{C 1} \\
\bar{R}_{\text {оп }}=\sum_{i=0}^{n}\left(\bar{R}_{i}+\bar{R}_{c i}\right) ; \\
\bar{R}_{\text {сп }}=\bar{R}_{\text {оп }}-\left[\sum_{i=0}^{n-1}\left(\bar{R}_{i}+\bar{R}_{c i}\right)+\bar{R}_{\text {п }}\right] .
\end{gathered}
$$

where $\bar{R}_{0}$ - radius vector of the top of the coordinate system $\mathrm{X}_{0} \mathrm{Y}_{0} \mathrm{Z}_{0}$ relative to the top of the coordinate system XYZ; $\bar{R}_{C 0}$ - radius vector of the center of the mass of the body $\mathrm{S}_{0}$ in the $\mathrm{X}_{0} \mathrm{Y}_{0} \mathrm{Z}_{0}$ coordinate system.

To determine the velocity vectors of the centers of inertia of the body relative to the appropriate coordinate system, you must perform the operation of differentiation with respect to time of the corresponding projections of the radius vectors at the axis of coordinate systems, as shown in $4 \mathrm{a}$ and $4 \mathrm{~b}$ in general case:

$$
\begin{aligned}
& \left\{\begin{array}{l}
\bar{V}_{00}=\bar{R}_{00}=\bar{V}_{0}+\bar{\omega}_{0} \times \bar{R}_{c o} ; \\
\bar{V}_{01}=\bar{R}_{01}=\bar{V}_{0}+\bar{V}_{1}+\bar{\omega}_{0} \times \bar{R}_{1}+\left(\bar{\omega}_{0}+\bar{\omega}_{1}\right) \times \bar{R}_{c 1} ; \\
\cdots \\
\bar{V}_{\text {оп }}=\bar{R}_{\text {оп }}{ }^{j}
\end{array}\right. \\
& \bar{V}_{i j}=\bar{R}_{i j}=\sum_{k=i}\left[\bar{V}_{k}+\left(\sum_{l=m}^{k} \bar{\omega}_{l}-\bar{\omega}_{k}\right) \times \bar{R}_{k}+\bar{\omega}_{k} \times \bar{R}_{c k}\right],
\end{aligned}
$$

where $\mathrm{i}=0,1,2, \ldots, \mathrm{N} ; \mathrm{j}=\mathrm{n}, \mathrm{n}+1, \ldots, \mathrm{N} ; \bar{V}_{k}=\bar{R}_{k}$ - velocity vector of pole $\mathrm{O}_{\mathrm{k}}$, associated with the body $S_{k}$ of the coordinate system $X_{k} Y_{k} Z_{k}$, relative to the coordinate system $X_{k-1} Y_{k-}$ ${ }_{1} Z_{\mathrm{k}-1}$, attached to the body $S_{\mathrm{k}-1}$.

Determination of the acceleration vector of ... centers of inertia of bodies Si respect to the corresponding coordinate systems is similar. We can show this in general case we get:

$$
\begin{aligned}
\bar{W}_{i j}=\dot{\bar{V}}_{i j}=\ddot{\bar{R}}_{i j}= & \sum_{k=i}^{j}\left\{\dot{\bar{V}}_{k}+2\left(\sum_{l=m}^{k} \bar{\omega}_{l}-\bar{\omega}_{k}\right) \times \bar{V}_{k}+\left(\sum_{l=m}^{k} \dot{\bar{\omega}}_{l}-\dot{\omega}_{k}\right) \times \bar{R}_{k}\right. \\
& +\left(\sum_{l=m}^{k} \bar{\omega}_{l}-\bar{\omega}_{k}\right) \times\left[\left(\sum_{l=m}^{k} \bar{\omega}_{l}-\bar{\omega}_{k}\right) \times \bar{R}_{k}\right]+\left[\left(\sum_{l=m}^{k} \bar{\omega}_{l}-\bar{\omega}_{k}\right) \times \bar{\omega}_{k}\right] \\
& \left.\times\left(\sum_{l=m}^{k} \bar{R}_{l}-\bar{R}_{k}+\bar{R}_{c k}\right)\right\}+\left(\sum_{l=m}^{k} \bar{\omega}_{k}\right) \times\left[\left(\sum_{l=m}^{k} \bar{\omega}_{k}\right) \times \bar{R}_{c j}\right],
\end{aligned}
$$

where $\dot{\bar{V}}_{j}$ - acceleration vector of the pole $\mathrm{O}_{\mathrm{j}}$, of the coordinate system $\mathrm{X}_{\mathrm{j}} \mathrm{Y}_{\mathrm{j}} \mathrm{Z}_{\mathrm{j}}$ attached to the body $S_{j}$ relative to the coordinate system $X_{j-1} Y_{j-1} Z_{j-1}$, attached to the body $S_{j-1}$ projected on the axis $X_{j} Y_{j} Z_{j}$.

The process of building a mathematical model of multitiered mechanical system is a sequence of operations presented in the form of the following algorithm (see Fig.3). 


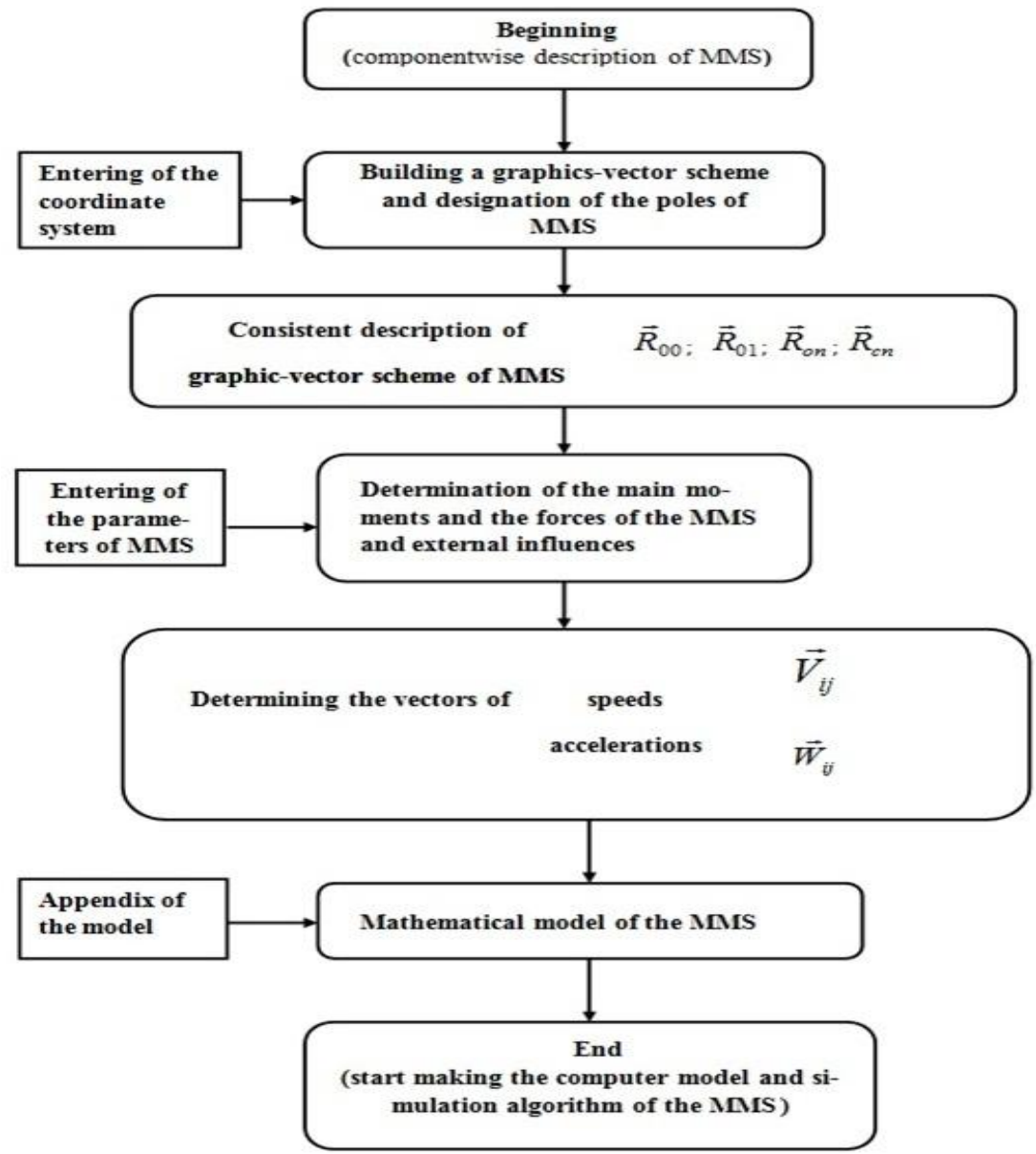

Fig. 3 Algorithm of building a mathematical model of the multitiered mechanical system

\section{CONCLUSION}

The technique of building a mathematical model of arbitrary multitiered mechanical system has been examined in this article. The relationship between the components of the system is determined using a graphic-vector scheme. Location of items in space is determined by the equations of the motion of a free body in vector form. The algorithm of building a mathematical model of the multitiered mechanical system has been also formed. The method is demonstrated on the example.

\section{CONCLUSION}

Ahmad, M., Alam, M., \& Talukder, M. (2013). Non-Associativity of Lorentz Transformation and Associative Reciprocal Symmetric Transformation. Asian Journal Of Applied Science And Engineering, 2(1), 48-56.

Ozada, N., \& Saeed Madani, S. (2012). A Novel Musculoskeletal Joint Modeling for Orthopaedic Applications. ABC Journal Of Advanced Research, 1(1), 35-46.

Talukder, M., \& Ahmad, M. (2013). Relativistic Rule of Multiplication of Velocities Consistent with Lorentz - Einstein Law of Addition and Derivation of the Missing Equations of Special Relativity. Asian Journal Of Applied Science And Engineering, 2(1), 26-41. 